Discussiones Mathematicae

\title{
CUBIC GRAPHS WITH TOTAL DOMATIC NUMBER AT LEAST TWO
}

\author{
SAieed Akbari \\ Department of Mathematics \\ Sharif University of Technology \\ e-mail: s_akbari@sharif.edu \\ Mohammad Motiei, Sahand Mozaffari \\ AND \\ SinA YAZDANBOD \\ Department of Computer Engineering \\ Sharif University of Technology \\ e-mail: motiei@ce.sharif.edu \\ smozaffari@ce.sharif.edu \\ syazdanbod@ce.sharif.edu
}

\begin{abstract}
Let $G$ be a graph with no isolated vertex. A total dominating set of $G$ is a set $S$ of vertices of $G$ such that every vertex is adjacent to at least one vertex in $S$. The total domatic number of a graph is the maximum number of total dominating sets which partition the vertex set of $G$. In this paper we provide a criterion under which a cubic graph has total domatic number at least two.
\end{abstract}

Keywords: total domination, total domatic number, coupon coloring.

2010 Mathematics Subject Classification: 05C15, 05 C69.

\section{REFERENCES}

[1] N. Alon and Z. Bregman, Every 8-uniform 8-regular hypergraph is 2-colorable, Graphs Combin. 4 (1988) 303-306.

doi:10.1007/BF01864169 
[2] B. Chen, J.H. Kim, M. Tait and J. Verstraete, On coupon colorings of graphs, Discrete Appl. Math. 193 (2015) 94-101.

doi:10.1016/j.dam.2015.04.026

[3] E.J. Cockayne, R.M. Dawes and S.T. Hedetniemi, Total domination in graphs, Networks 10 (1980) 211-219. doi:10.1002/net.3230100304

[4] T.W. Haynes, S.T. Hedetniemi and P.J. Slater, Fundamentals of Domination in Graphs (CRC Press, 1998).

[5] T.W. Haynes, S.T. Hedetniemi and P.J. Slater, Domination in Graphs: Advanced Topics (Marcel Dekker, 1998).

[6] M.A. Henning and A. Yeo, Total Domination in Graphs (Springer, 2013). doi:10.1007/978-1-4614-6525-6

[7] M.A. Henning and A. Yeo, 2-Colorings in k-regular $k$-uniform hypergraphs, European J. Combin. 34 (2013) 1192-1202. doi:10.1016/j.ejc.2013.04.005

[8] J. Spencer, Asymptotic lower bounds for Ramsey functions, Discrete Math. 20 (1977) 69-76. doi:10.1016/0012-365X(77)90044-9

[9] J. Spencer, Ten Lectures on the Probabilistic Method (SIAM, 1987).

Received 26 February 2016

Revised 28 September 2016 Accepted 28 September 2016 